\title{
Nuclear Electric Vehicle Optimization Toolset (NEVOT)
}

\author{
Michael L. Tinker ${ }^{*}$, James W. Steincamp, Eric T. Stewart, Bruce W. Patton, William P. Pannell, Ronald L. Newby, \\ Mark E. Coffman, and Larry D. Kos \\ NASA Marshall Space Flight Center, Huntsville, Alabama, 35812 \\ A. Lou Qualls and Sherrell Greene \\ Oak Ridge National Laboratory, Oak Ridge, Tennessee \\ and \\ Steve Bancroft and Greg Molvik \\ Arnold Engineering Development Center, Arnold Air Force Base, Tennessee
}

\begin{abstract}
The Nuclear Electric Vehicle Optimization Toolset (NEVOT) optimizes the design of all major nuclear electric propulsion (NEP) vehicle subsystems for a defined mission within constraints and optimization parameters chosen by a user. The tool uses a genetic algorithm (GA) search technique to combine subsystem designs and evaluate the fitness of the integrated design to fulfill a mission. The fitness of an individual is used within the GA to determine its probability of survival through successive generations in which the designs with low fitness are eliminated and replaced with combinations or mutations of designs with higher fitness. The program can find optimal solutions for different sets of fitness metrics without modification and can create and evaluate vehicle designs that might never be considered through traditional design techniques. It is anticipated that the flexible optimization methodology will expand present knowledge of the design trade-offs inherent in designing nuclear powered space vehicles and lead to improved NEP designs.
\end{abstract}

\section{Introduction}

$\mathrm{T}$ HE integration issues associated with using reactor power for actual space science missions have received little attention to date and the design trade-offs that must occur on a reactor-powered space vehicle are not fully known. The Nuclear Electric Vehicle Optimization Toolset (NEVOT) is designed to uncover the unknown trades so they can be studied and understood. NEVOT is a nuclear electric propulsion (NEP) vehicle optimization algorithm based on a genetic algorithm (GA) search technique. The tool searches for the optimal vehicle subsystem combinations to perform user defined missions.

Traditional methods of multi-disciplinary system design are sequential in nature. A subsystem is selected and designed, and inputs from the first subsystem design are passed to the "next" subsystem, which is sized or designed to be compatible with the first subsystem. The process is repeated until all of the subsystems are designed. The entire system is then compared to known constraints and compromises are made between subsystems to meet those constraints. Once a base design is complete, the system is perturbed in an effort to optimize it for a desired result, such as minimizing mass or cost. Automated sequential multi-disciplinary design efforts typically mimic human designers and are sequential in nature. The resulting configurations are a strong function of the information design logic built into them.

NEVOT attempts to remove potentially limiting foreknowledge from the design process in order create subsystem arrangements that might never result from traditional design methods, whether automated or not. By removing the trends that are inherently designed into complex multi-disciplinary systems we can hopefully uncover new trends that will lead to improved designs. The tool allows for the combination of tens of thousands of subsystem designs without regard to their compatibility or combined ability to meet the mission requirements. The ability of each combination of subsystems to perform a mission is evaluated after they are created and assigned a

\footnotetext{
*Aerospace Technologist; Structures, Mechanics and Thermal Department; ED20; Associate Fellow AIAA.
} 
figure of merit, called "fitness". The fitness of each vehicle is used within a genetic algorithm to search the possible combinations to find the best vehicles to perform the mission.

Genetic algorithms comprise a type of non-gradient-based search technique that mimics biological adaptation and reproduction to produce improvement in designs from generation to generation, until the fittest solutions are produced. The basic idea behind the genetic algorithm is to define a representation of the system, sometimes referred to as a chromosome. Each chromosome is a collection of variables, or genes, that describe the vehicle. Each gene is allowed to vary within a range representing legitimate engineering choices. The type of information encoded into a gene can include the type or material of a component, the number of the components, the size or rating of components, the operating temperature of a component, among others.

Within the GA, the first generation of vehicles is created by combining randomly-created subsystems. The fitness of each vehicle to meet the mission requirements is then evaluated. Vehicle designs with higher fitness values are allowed to remain in the population and the remaining designs are removed from the population. The population is returned to its original size by (1) adding combinations (children or offspring) of the more fit designs, (2) adding new designs created from fit designs with a single characteristic altered (mutations), and (3) adding new designs that are "clones" of the better designs. Through successive generations, the fittest population of vehicles within the solution space will remain. By including optimization parameters, such as mass or cost, in the fitness evaluation, the vehicles that meet the mission requirements (constraints) while minimizing or maximizing the parameter(s) of interest can be found.

The independent creation of subsystem designs can lead to combinations of subsystems that simply do not make sense. These combinations will have a low fitness and will be eliminated from the population. However, the concept of independent creation also leads to combinations that have never been thought of before, or are precluded by logic encoded within a sequential design process. By studying the surviving specimens, the design trade-offs that occur within the population to make better designs can be observed and understood. Evaluation of the common characteristics of the optimal solutions can lead to (a) identification of those characteristics most important to the mission objectives and (b) understanding of interactions of the characteristics to produce more optimal solutions.

\section{Vehicle Component Modeling}

In NEVOT, a space reactor power system (SRPS) is combined with a spacecraft configuration module, a power management and distribution module, a truss structure module, an electric propulsion (EP) module, and a trajectory module. The modules are used to take the limited vehicle description contained in the chromosome, complete the vehicle design, and evaluate the vehicle's ability to perform a defined mission. The reference mission is defined by the destination, time on station, target mission duration and payload. Each subsystem design module is to take a limited set of input variables (some varied by the GA and others fixed), finish a more detailed design of the subsystem (if necessary) and return as output (1) information required to evaluate the subsystem's ability to work with other subsystems, (2) information required of all subsystems to evaluate the system, and (3) an estimation of parameters of interest that are to be optimized by the algorithm (such as mass).

\section{A. Subsystem Module Development}

The development of subsystem modules was initially limited to simple simulation models (so-called Level 0 models) to allow the integration methodology and code interfaces to be developed and successfully demonstrated. Most of the modules simply consist of several pages of FORTRAN, with the exception of the PMAD module. It was desired to have one relatively detailed module (Level 1 model) as part of the initial feasibility demonstration phase. It is noted that the Sandia Laboratories' DAKOTA software was chosen for optimization of the subsystems, and the implementation will be described in detail in a separate section of the paper. Each of the subsystem modules is described next in this section.

\section{Space Reactor Power System Module}

The first generation SRPS module is an integrated low-level FORTRAN module that models a lithium-cooled pin type reactor either directly or indirectly coupled to a potassium Rankine power conversion system. The module uses relatively simple scaling relations to size the reactor, shield, and power conversion components in a manner that minimizes overall SRPS mass within the constraints defined by the fixed variables in the input file. 


\section{Spacecraft Configuration Module}

The configuration module generates geometric information for the spacecraft. The initial version is simplified to a minimum degree of complexity and assumes a spacecraft configuration that is somewhat consistent with various robotic nuclear missions studied recently. The artificial gravity is achieved by rotating about the long axis of the spacecraft. The configuration and defining variables are shown in Fig. 1.

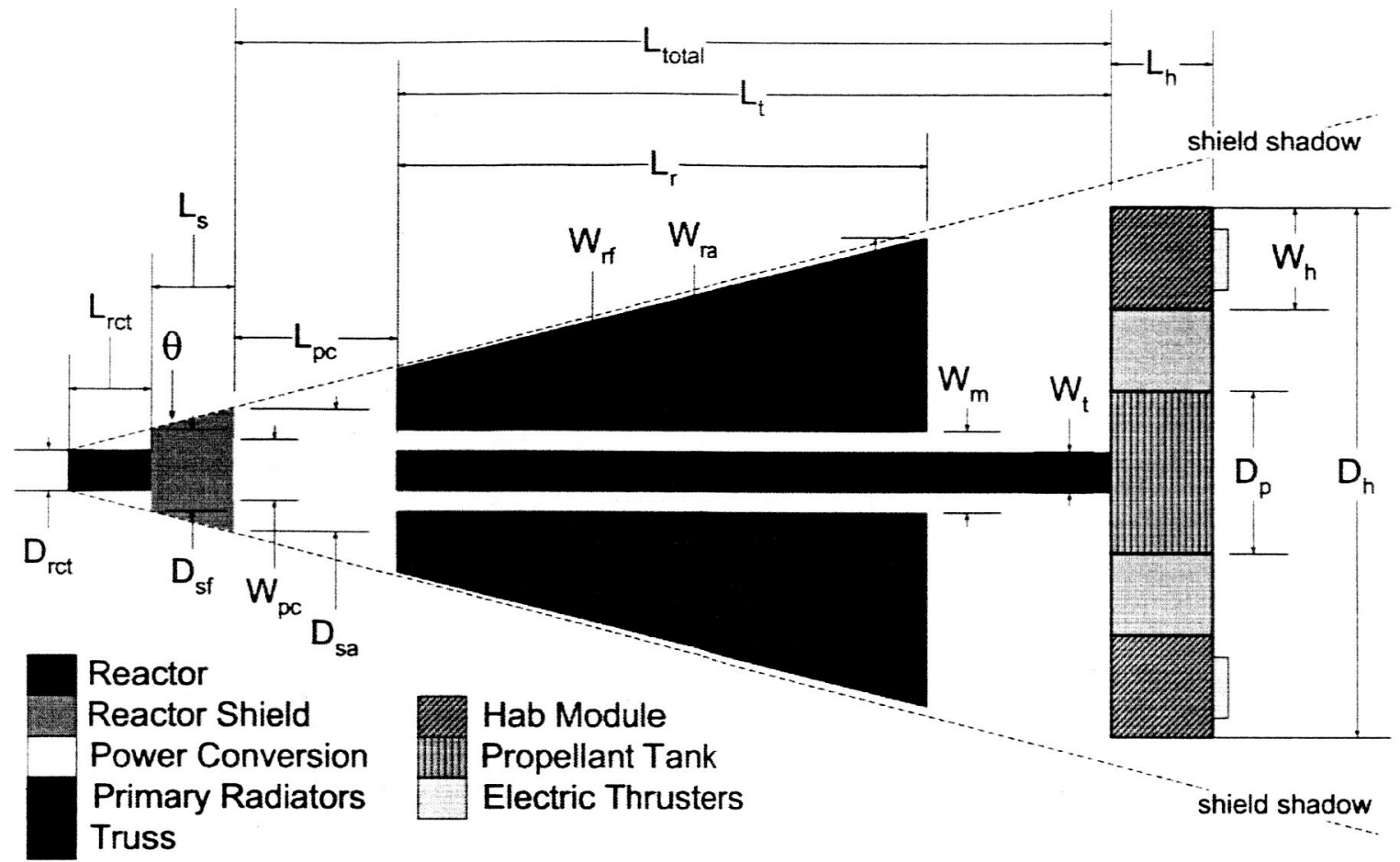

Figure 1. Initial NEVOT configuration and the defining variables.

In addition to the basic configuration, the driving assumptions are that; 1) all hardware will remain within the shield shadow, 2) the radiators are adjacent to the power conversion section, and 3 ) the cross-section of the reactor shield is circular. Basically, the configuration module takes the DAKOTA-defined radiator area, hab module diameter, shield thickness, forward shield diameter, and shield half-angle, then calculates the minimum required truss length to fit everything within the shield shadow. The minimum required truss length is compared to the DAKOTA-defined truss length, and the DAKOTA-defined spacecraft is penalized appropriately. An analogous penalty is also performed on the forward shield diameter based on the reactor diameter and length determined by the SRPS module. A useful by-product of the configuration module is the output of files that can be used to visualize the configuration.

As previously stated, the current version of the configuration module contains many simplifying assumptions. In addition, many of the variables shown in Fig. 1 were fixed so that the focus could reside on the integration of the various modules within DAKOTA. Continuation of NEVOT will focus both on the relaxation of assumptions and on allowing the integrated variation of additional variables. For example, presently the power conversion volume is an input based on an extremely rough scaling from lower power robotic missions. The length and width of the power conversion section are adjusted by the configuration module, which insures that this section remains within the shield shadow and conserves the initial volume. This approach to defining the power conversion geometry is certainly a prime candidate for improvement. Additional candidates include: 1) variation of the hab module geometry to capture the integrated effects of the artificial gravity magnitude and required rotational velocity, 2) inclusion of additional radiator shapes (e.g., stepped, rectangular, etc.), and 3) accounting for required thruster area in comparison with available area at the back of the hab module. As a final note, the configuration module does not presently compute the centers of mass of the various subsystems and components. This capability will also be added within the framework of the existing geometric simplifications. 


\section{Power Management and Distribution (PMAD) Module}

The PMAD code is composed of approximately fifty pages of FORTRAN 77, thus it is more detailed than the other modules. It was desired during the early phase of the project to demonstrate the capability of at least one Level-1 module during the first year. The PMAD module uses the FOTRAN based-code developed by Rocketdyne $^{(1)}$ for NASA as part of the Space Exploration Initiative program. The architecture is based on the use of an $\mathrm{AC}$ power transmission at a designated alternator voltage and frequency, which was determined from the basis of a comparison studies ${ }^{(2)}$ between $\mathrm{AC}$ and DC transmission and low frequency versus high frequency. The lowfrequency PMAD model provides overall system mass and efficiency information. It can model different alternator and thruster types and it can accept power levels ranging from $10 \mathrm{Kwe}$ to $100 \mathrm{Mwe}$, transmission voltages ranging from 1000 to $10,000 \mathrm{Vrms}$, and alternator frequencies ranging from $60 \mathrm{~Hz}$ to $5 \mathrm{Khz}$. The code allows the generation of PMAD components for three different thruster types: (1) ion engine w/o transformer, (2) ion engine with transformer, and (3) MPD thrusters. The subroutines model various aspects of the alternator speed regulator, alternator-switchgear transmission line, phase lock transformer, switchgear, switchgear-switchgear transmission line, switchgear-thruster transmission line, shunt regulator-parasitic load radiator transmission line, and the parasitic load radiator. A limited number of the available input variables were selected for use by the genetic algorithm, and the remaining variables used the code's internal default values.

\section{Truss Module}

A simple first-principles truss module was written in FORTRAN code for the initial development program. The module has as genes the material (limited to aluminum), number of long (main) members (3 to 5), outer diameter and wall thickness of the main members, and length of the main members. An approximate mass calculation is made by first computing the long member masses, then adding a 20 percent contribution for cross members. A simplified buckling load calculation is accomplished by (a) dividing the total applied axial load by the number of long (main) members, and (b) computing the critical Euler buckling load based on the length and cross-sectional properties of the main members. It is noted that improvements will be made to this module to include the effects of the cross members in the buckling calculations, and to more accurately account for mass of cross members. As the vehicle optimization tool matures, the truss module will be converted from simple code to a NASTRAN finite element model with interface code to DAKOTA.

\section{Electric Propulsion Module}

The electric propulsion (EP) module is a FORTRAN code in which the type of thrusters on the vehicle is selected, as are the type and mass of the propellant. Currently, ion, Hall, and MPD thrusters are provided in the code. The EP and tank module calculates the mass flow rate, number of thrusters, total thrust, and total mass of the EP subsystem. For each thruster type, the module provides values for Isp, maximum thruster diameter, thrust per unit area, thrust per unit mass, and thruster efficiency. The module utilizes only system-level computations from historical databases and rules of thumb.

\section{Trajectory Module}

Figure 2 shows the defined reference mission (to the asteroid belt) that is used as a base case for development purposes. The manned vehicle is to leave low earth orbit, travel approximately $4 \mathrm{AU}$, remain on station for 30 days and return to low earth orbit. A trajectory analysis was performed in VariTOP resulting in an optimized electrical power for a fixed range of Isp values, a fixed range of trip times, and a single fixed power and propulsion system alpha. The trajectories are shown in Figure 2. The traces in bold black indicate periods of thrust during the mission.

Within the trajectory module each vehicle's total electrical power, the thruster Isp, and total vehicle mass are used to perform a table look-up into the VariTOP data, which results in a corresponding estimate of trip time. To provide greater fidelity for future efforts, the VariTOP analysis will be expanded to include a greater range of Isp values, a greater range of trip times, and a greater range of alpha (ratio of vehicle dry mass to thruster electrical power) values. 

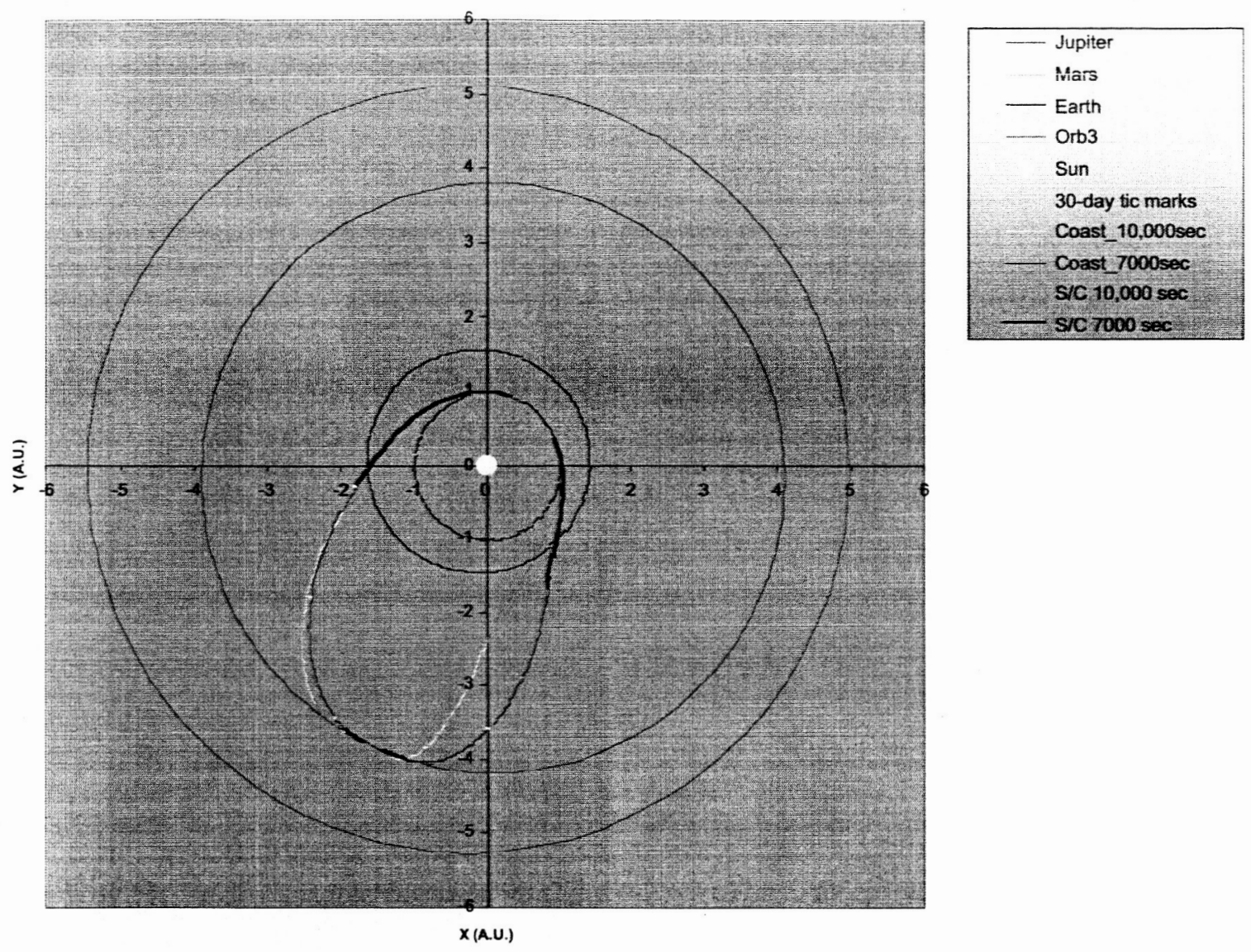

Figure 2. Trajectories for reference mission to $4 \mathrm{AU}$.

\section{B. Example Vehicle Configuration}

Much initial effort was focused on defining the interfaces between the modules and the optimization code to allow seamless replacement of low-fidelity models with more detailed and sophisticated models as they are developed. An example vehicle configuration, shown in Fig. 3, was established to permit the development of the evaluation algorithm. The reactor and shield are upper left in the figure and are extended from the habitat (green) by a truss system. The radiator panels (red), power conversion systems and PMAD systems fit between the shield and the habitat. This vehicle spins about its main (longitudinal) axis to produce and acceleration field at the outer radius of the habitat.

It is noted that it is not the purpose of the NEVOT project to design a particular nuclear electric vehicle, but to develop a set of optimization tools that can be used in the future for that purpose. Thus an actual manned nuclear vehicle would likely be much different that what is shown conceptually in Fig. 3. 


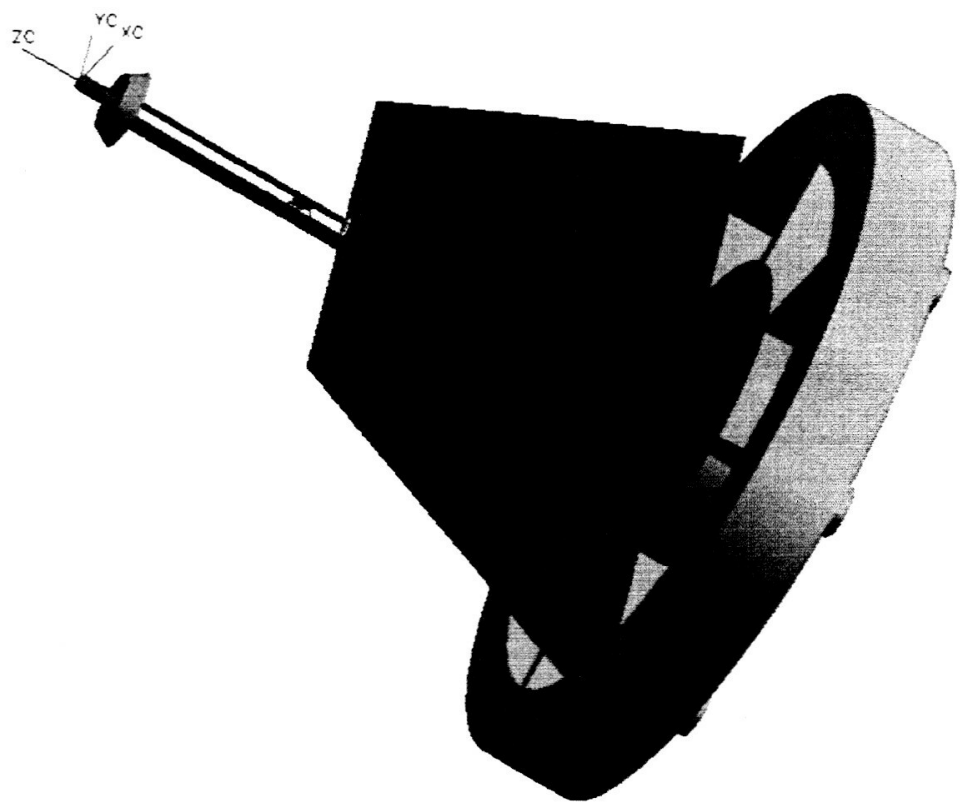

FRONT WORK

Figure 3. Example conceptual configuration of the NEP vehicle being optimized by NEVOT as developed by Marshall Space Flight Center.

\section{Implementation of Genetic Algorithm-Based Optimization}

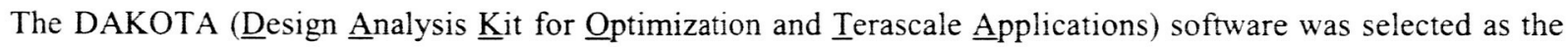
optimization tool to apply the GA technique to the NEVOT programs. DAKOTA is a multilevel parallel object oriented framework for design optimization, parameter estimation, uncertainty quantification and sensitivity analysis. It contains an extensive set of gradient based and non-gradient based optimization techniques, which makes it possible to perform direct comparisons between to two techniques.

The relationship among DAKOTA, the evaluation programs and the fitness evaluation program are shown schematically in Figure 4. The DAKOTA GA algorithm randomly generates an initial population of vehicles and manipulates the vehicle description input file so that NEVOT can assess the fitness of each "created" vehicle. The fitness assigned to an individual is used in the GA algorithm to determine its worth within the population and therefore its probability of survival. Crossovers and mutations from fit vehicles are randomly generated to see if more fit individuals might be created. It is possible, and indeed more likely than not, that a vehicle's subsystems will not be well matched to one another. There is no penalty for this except that the system as a whole receives a low fitness and is bred out of future populations. After many generations only the fittest vehicles remain.

There are three major user-defined components in vehicle design and optimization under NEVOT: the selection of a set of vehicle description variables that define the chromosome (or individual) varied by the GA, the selection of the vehicle fitness function, and the selection of parameters that define the behavior of the GA. In order to establish initial proof of concept, a limited set of vehicle design variables was defined and a single vehicle fitness function was developed. A brief description of the user-defined components follows. Representative results are shown in the next section.

Of the 48 unique inputs to the initial NEVOT subsystem modules, 18 were selected as elements in the set of vehicle description variables varied by the GA, 27 were assigned constant values, and 3 were derived from subsystem outputs. A list of the inputs varied by the GA and their respective ranges are given in Table 1 . 


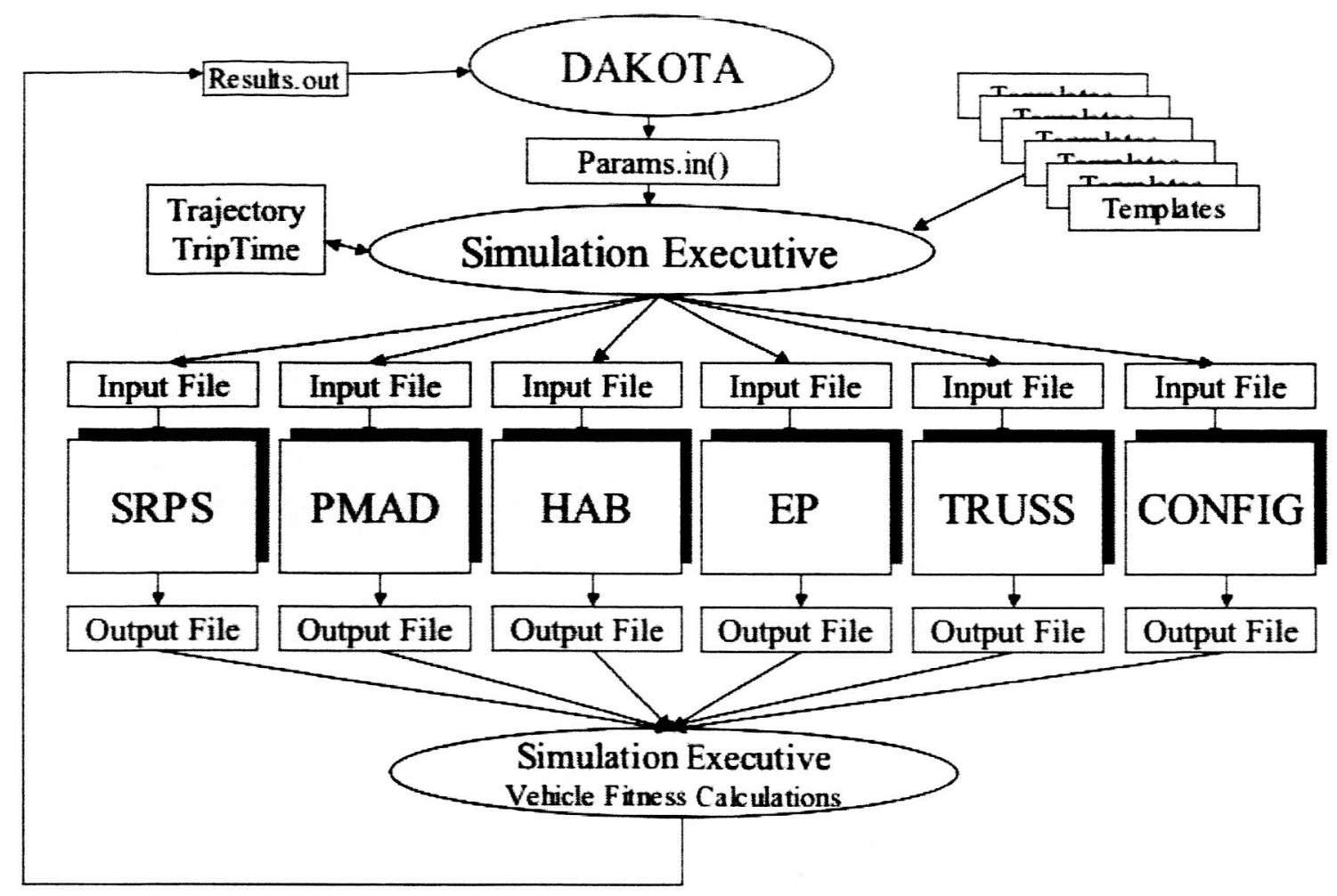

Figure 4. Schematic representing relationships of the components of NEVOT.

Table 1. Initial description variables in the vehicle's chromosome.

\begin{tabular}{|l|l|l|}
\hline Variable & Minimum Value & Maximum Value \\
\hline Total electrical power for vehicle (W) & 3490000.0 & 15600000.0 \\
\hline Cone Angle (degrees) & 0.0 & 80.0 \\
\hline Front shield diameter (m) & 0.0 & 5.0 \\
\hline Neutron shield thickness (m) & 0.0 & 5.0 \\
\hline Gamma shield thickness (m) & 0.0 & 5.0 \\
\hline Total radiator area (m^2) & 100.0 & 5000.0 \\
\hline Truss boom length (m) & 20.0 & 250.0 \\
\hline Number of truss long members & 3 & 5 \\
\hline Truss long member outer diameter (cm) & 1.0 & 100.0 \\
\hline Truss long member wall thickness (mm) & 0.318 & 31.8 \\
\hline Power processing unit alternator operating frequency (kHz) & 0.06 & 5.0 \\
\hline Power processing unit input voltage (V rms) & 200.0 & 10000.0 \\
\hline Power processing unit transmission line length (m) & 25.0 & 200.0 \\
\hline Propellant mass (kg) & 1500.0 & 100000.0 \\
\hline Pressure vessel material index & 1 & 4 \\
\hline Turbine material index (1-Ni, 2-Ta) & 1 & 2 \\
\hline Number of turbines operating & 2 & 4 \\
\hline Thruster type, ISP, and Power processing unit index & 1 & 3 \\
\hline
\end{tabular}


The vehicle fitness function focused both on minimizing vehicle mass and on penalizing vehicle designs that did not meet real-world constraints. The function $\mathrm{f}^{\prime}(\mathrm{x})$ was implemented as a summation of vehicle mass $f(x)$ and nine weighted constraints, as shown in Eq. (1):

$$
f^{\prime}(x)=f(x)+\sum_{n=1}^{9} g_{n}(x)
$$

The constraints consisted of trip time, fuel remaining, truss length, front shield diameter, neutron shield thickness, gamma shield thickness, alpha, force applied to truss, and radiator area. Each of these parameters where provided by the vehicle chromosome to the modules. The modules also calculate each of the nine parameters to be used as verification that the resulting vehicle chromosome actually meets the mission criteria. For example, the GAgenerated parameters are provided to the configuration module that calculates a truss length. The configuration module ensures that the calculated truss length is long enough to keep the habitat module and other vehicle components behind the shadow of the reactor's shield. If the GA-provided truss length is less than the truss length calculated by the truss module, then this vehicle design will be penalized. The constraint for the truss length $g_{1}(x)$ is calculated as follows:

$$
g_{1}(x)=\left(1-\left|\frac{\text { truss length }_{\text {calculated }}}{\text { truss lengthGA }_{G}}\right|\right) \times f(x)
$$

Also, each of the other constraints are calculated in a similar manner:

$$
g_{n}(x)=\left(1-\left|\frac{\text { constraint } \text { parameter }_{\text {calculated }}}{\text { constraint parameterGA }}\right|\right) \times f(x)
$$

The real-valued genetic algorithm from DAKOTA's Stochastic Global Optimization library was selected for use as the GA. The parameters selected for the GA were: population size of 50 individuals, upper limit of 20000 iterations, upper limit of 2000 function evaluations, the use of the two-point crossover method for new chromosome generation, and the use of the elitist selection method for new population selection. After the initial random creation of a population, the elitist method would select individuals for each successive population from a fixed number of best individuals from the current population and a fixed number of randomly chosen new individuals. The new individuals were created by the two-point crossover method, which combined genetic material from two parents in a coordinate-wise fashion. Each parent was split into three regions, with the new individual created from the middle region of one parent and the end regions of the other.

\section{Initiai Optimization Resuits}

The objective for initial NEVOT efforts was to develop and demonstrate a framework for integrating vehicle subsystems and performing design and optimization using a GA. To aid in the integration efforts, several of the initial subsystem modules were limited in fidelity. As such, the vehicle design results presented in this section are preliminary and may not accurately reflect state of the art design. However, plans for second year efforts include greatly increasing the fidelity of all of the subsystem modules. Results are provided to demonstrate some of the capability of the NEVOT tool. Figure 5 provides a geometric view depicting 2 of the 2000 iterations from the vehicle chromosome. Figure 6 provides a geometric view and resulting parameters of the initial optimized vehicle. 


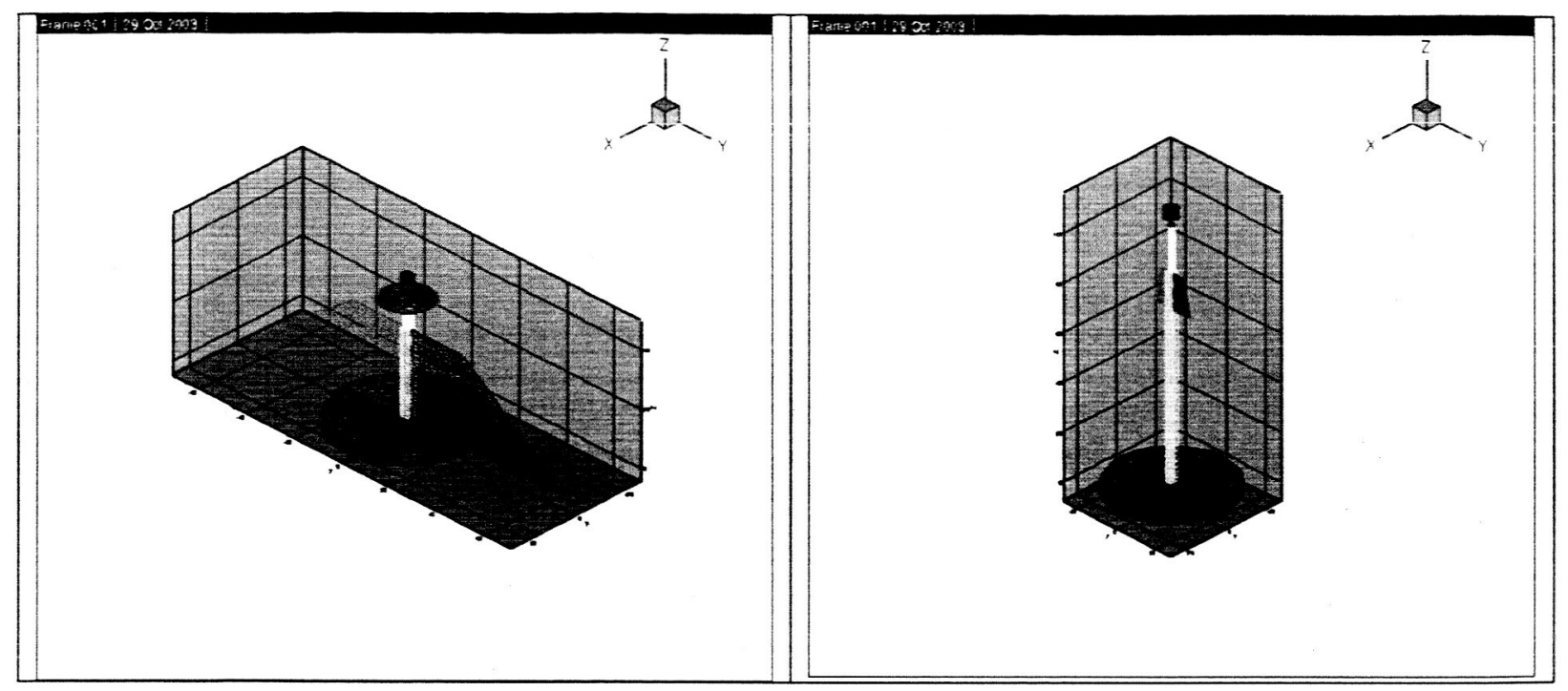

Figure 5. Geometric View of Two Sample Design Iterations.

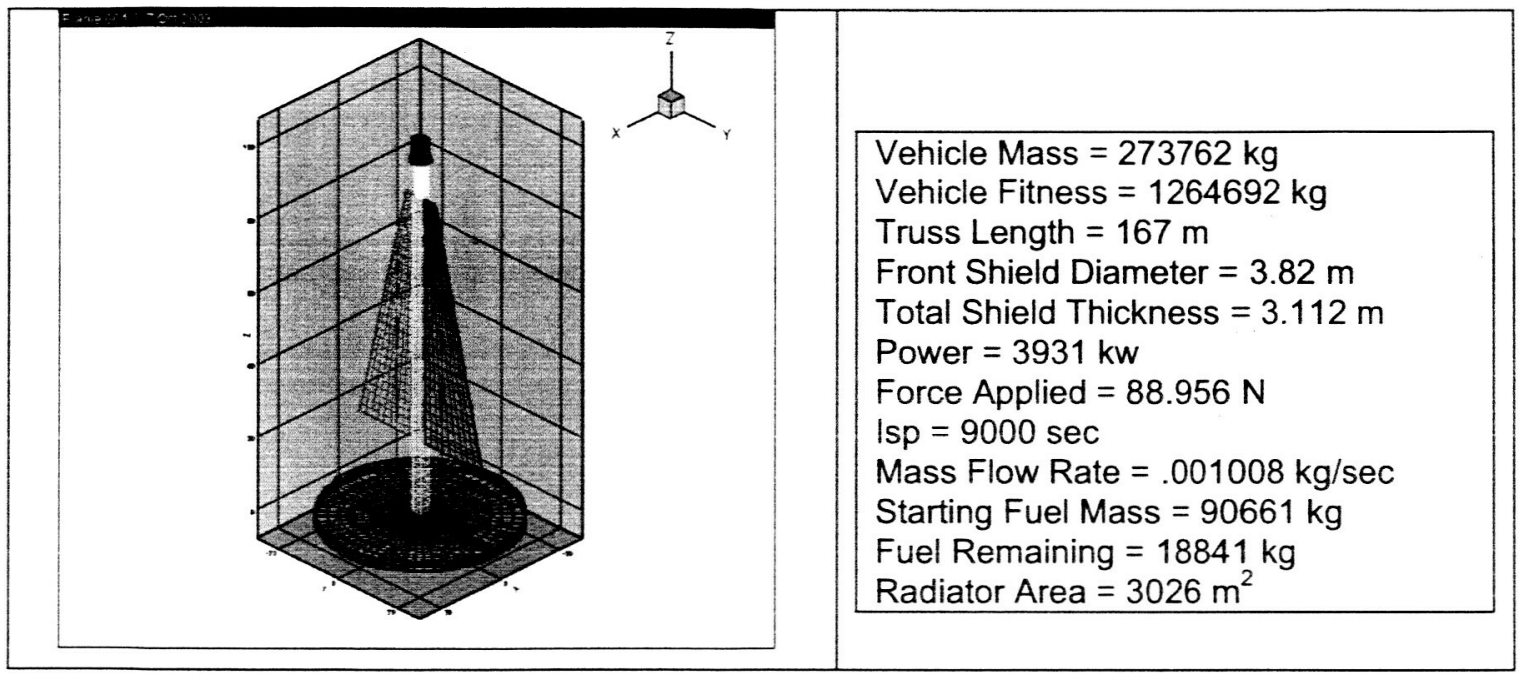

Figure 6. Geometric view and parameters for the optimized vehicle.

\section{Summary}

The power of the NEVOT methodology is that combinations of subsystems are not logically coded together. The GA algorithm will experiment with truly unique vehicle combinations, which provides the power to find new and better solutions. Further, once the algorithm is developed it does not have to be modified to search for the optimal solution to different missions, to emphasize a different set of optimization parameters or to use a different definition of fitness. This makes NEVOT a flexible and powerful tool for performing subsystem trades studies.

\section{References}

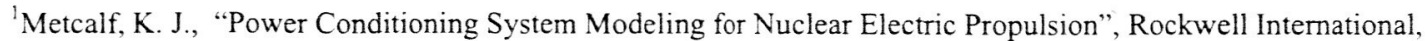
Rocketdyne Division, NASA-CR-191136, Canoga Park, CA, Nov. 1993.

${ }^{2}$ Metcalf, K. J., “ Multimegawatt Dynamic NEP PMAD Study”, Tenth Symposium of Space Nuclear Power and Propulsion, Albuquerque, NM, 1993. 